\title{
Research Article \\ Stability Control of Vehicle Emergency Braking with Tire Blowout
}

\author{
Qingzhang Chen, Youhua Liu, and Xuezhi Li \\ College of Automobile Engineering, Changshu Institute of Technology, Changshu 215500, China \\ Correspondence should be addressed to Qingzhang Chen; chenqz1973@sina.com
}

Received 1 October 2013; Revised 19 December 2013; Accepted 28 January 2014; Published 11 March 2014

Academic Editor: Rakesh Mishra

Copyright ( 2014 Qingzhang Chen et al. This is an open access article distributed under the Creative Commons Attribution License, which permits unrestricted use, distribution, and reproduction in any medium, provided the original work is properly cited.

For the stability control and slowing down the vehicle to a safe speed after tire failure, an emergency automatic braking system with independent intellectual property is developed. After the system has received a signal of tire blowout, the automatic braking mode of the vehicle is determined according to the position of the failure tire and the motion state of vehicle, and a control strategy for resisting tire blowout additional yaw torque and deceleration is designed to slow down vehicle to a safe speed in an expected trajectory. The simulating test system is also designed, and the testing results show that the vehicle can be quickly stabilized and kept in the original track after tire blowout with the emergency braking system described in the paper.

\section{Introduction}

Tire blowout is one of the main factors which cause the fatal traffic accidents. Currently, the tire pressure monitoring system (TPMS) is popularly equipped on vehicles to prevent low tire pressure and avoid tire failure accidents. But it can only remind the driver of slow tire pressure failure [1].

For the situation of urgent tire blowout, the characteristics of vehicle motion will be changed after tire blowout. It is easy to cause the driver's rush irritability operation [2]; once the driver is operating improperly, the vehicle will be severely side slipped, drifted, and even overturned. So it is useful to develop an emergency braking system to slow down the velocity of the vehicle automatically and smoothly [3-5].

A tire blowout automatic braking system is developed, which can slow down the vehicle automatically to a safe speed and stop the vehicle before going out of the driver's control. By using this system, it can offset the driver's delayed braking and help the tire-failed vehicle slow down safely.

\section{Tire Failure Emergency Braking System}

The emergency braking system for tire blowout is shown in Figure 1. Based on the electrohydraulic braking system
(EHB), a yaw rate signal from the yaw rate sensor and the tire blowout signal input are added. The automatic braking is realized by the control unit driving the brake motor pump and each related electromagnetic valve to brake after receiving the tire blowout signal. According to the yaw rate and the deviation, the braking state of the vehicle is determined, then the pressure of each cylinder is determined, and the related differential braking is carried out to control the vehicle moving stability. With the wheel speed signals, the slip rate of each wheel is calculated, and the related wheel cylinder valves are driven to carry out antilock brake control.

The actuators of the system are shown in Figure 2. It is made up of conventional brake, the hydraulic power supply parts of the automatic braking, inlet and outlet valves of each wheel cylinder, separating valves, and balance valves. During the normal moving of the vehicle, the automatic hydraulic braking pipe maintains certain prepressure under the function of the accumulator. When in the conventional braking, brake pedal is working, and the hydraulic pressure is transferred from the separating valve and balance valve to each wheel cylinder to obtain braking force (the pedal feeling simulator and corresponding parts are not shown in the EHB system). Once there is a tire blowout, the automatic braking system is activated. With the working of the separating valves, 


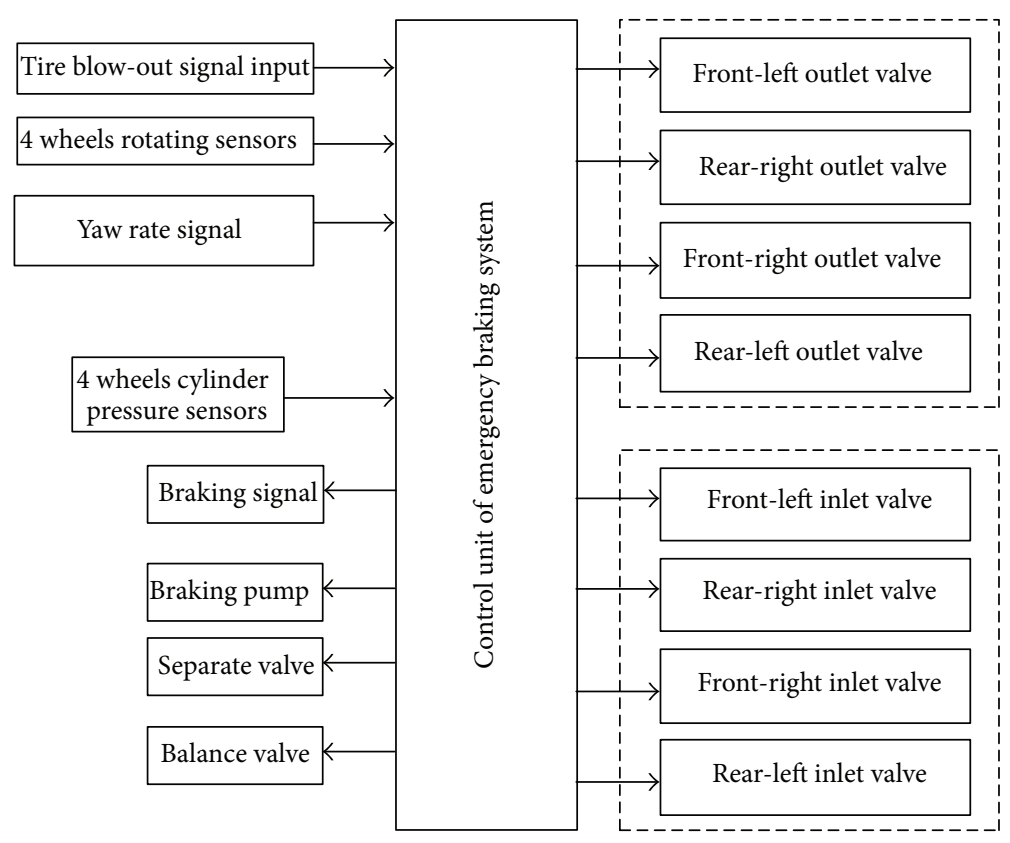

FIGURE 1: System hardware principle.

balance valves, and the opening of the inlet valves, braking pressure is transferred from the hydraulic power supply to each wheel cylinder. According to the vehicle yaw rate signal, the controller of the emergency automatic braking system judges the operation condition and then adjusts the amplitude of the pressure in each wheel cylinder to realize the differential braking and control the moving trajectory of the vehicle.

\section{The Strategy of the System}

3.1. The Module of Automatic Braking. At the moment of the tire bursting, the yaw movement of the vehicle produced by tire blowout not having been established, the four wheels are working with braking force, and the duration time is $t_{0}$. Then, the additional yaw torque which is produced by the tire failure is becoming larger, and the vehicle is off its original trajectory. According to the additional torque, the antiyaw torque which is provided by differential braking to balance the tire blowout additional one is evaluated. The system boosts the two of the diagonal wheel cylinders offside of the failed tire $[7,8]$. According to the yaw rate signal of the vehicle, the deviation value of the vehicle movement can be confirmed. If the deviation is anticlockwise then the value would be positive and vice versa. If the value is in the threshold, braking force can be enhanced in the four-wheel cylinders at the same time. If yaw rate is positive and exceeding the threshold, then a resistant clockwise torque is executed by differential braking. If yaw rate is negative, then a resistant anticlockwise torque is executed. The normal ABS is activated every $50 \mathrm{~ms}$, once there is a need of ABS, and is prior to the emergency stability control. Once the vehicle speed drops to $20 \mathrm{~km} / \mathrm{h}$, the vehicle keeps the original braking state till it stops.
Figure 3 shows the controlling procedure for vehicle emergency braking with tire blowout (only flag $==3$ is showed, which means left-rear wheel failure). Here, $z_{0}$ is the needed intense threshold of the emergency braking. $\Delta$ is the deviation threshold of yaw rate. $e$ is the deviation of yaw rate. The failure of other wheels is similar to it, and the only thing is to rectify the flag of the corresponding tire blowout signal.

When the left-rear wheel is detected to be tire failed, the system increases 4-wheel cylinders' braking pressure, that is, the state values shown in Figure 4 (a-LF means the control state value for left-front tire failure, $\mathrm{b}-\mathrm{RF}$ is for rightfront tire failure, c-LR is for left-rear and d-RR is for rightrear tire failure) being state3.LR_state $=3$, to gain a light braking on whole and then carry out state3.LR_state $=1$, to gain an antitire blowout yaw torque. $t$ in Figure 3 means the estimated holding time of the state value. After state3, the system performs the vehicle braking stability process of other state values according to the threshold of yaw rate deviation $e$ and deceleration $\dot{v}$, that is, $\Delta$ and $z_{0} g$.

The implementation state values of the emergency braking of the failed tire are shown in Figure 4(a), (b), (c), and (d). There are four kinds of tire failure (only one tire failure is discussed in this paper) and 16 states. As shown in Figure 4, FS and RS stand for the state value of the front and rear separating valves, respectively, and FS = 1 and RS = 1 mean that the separating valve is working; that is to say, it is separated. FB and RB stand for the state values of the front and rear balancing valve, respectively. As shown in the diagrams, if the "statel.LF/RF/LR/RR_state" value is 0 , then the pressure of each wheel cylinder is kept at a certain level; if the value is 1 , then the anticlockwise yaw torque is performed; if the value is 2 , then the resistance anticlockwise yaw torque is performed; if the value is 3 , then all of the four wheels of the vehicle are performed the same braking 


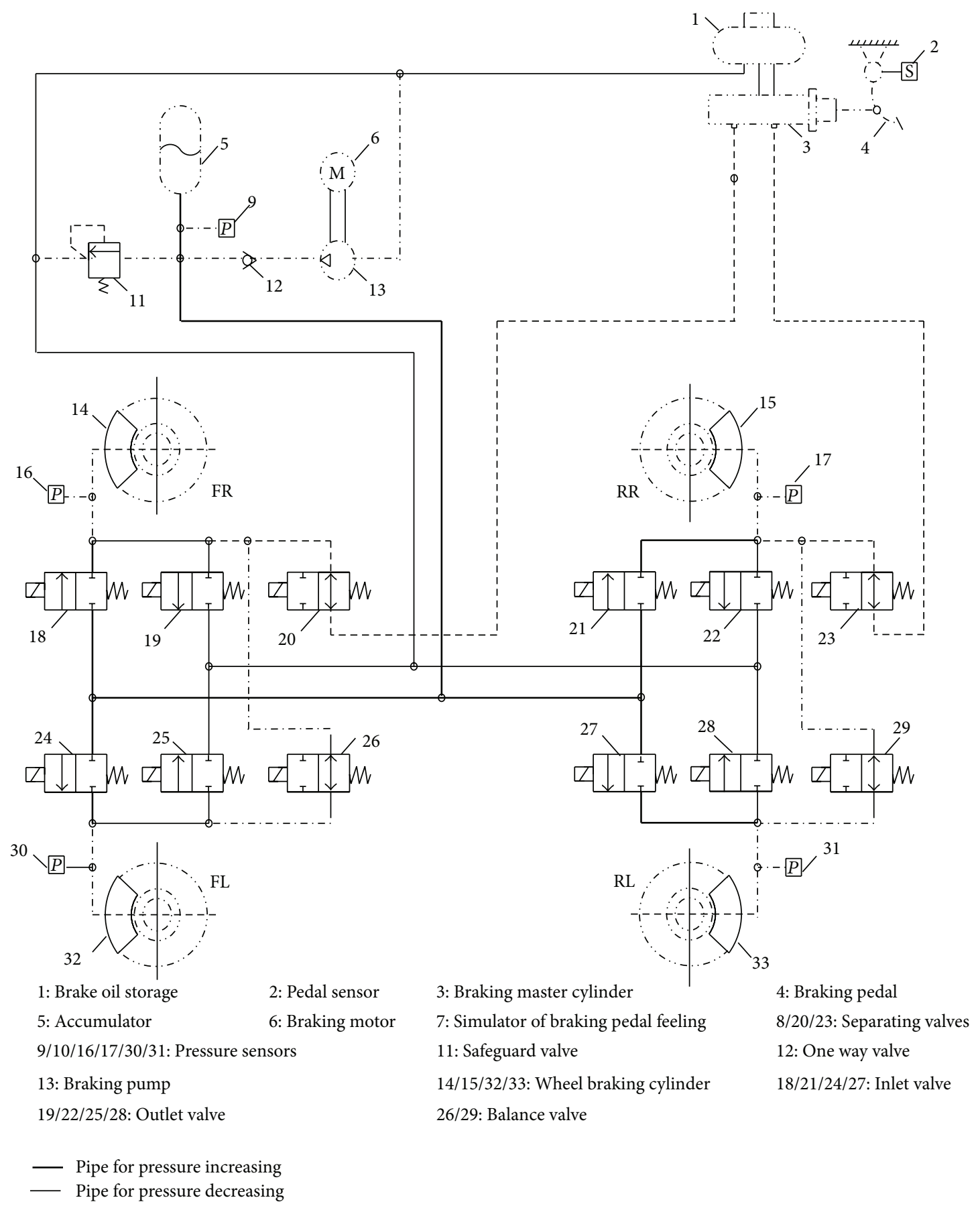

Figure 2: The actuators of the system [6].

force. LF_in, out $=0,1$ stand for left-front wheel cylinder inlet and outlet valves close or open; RF_in, out $=0,1$ stand for front-right wheel cylinder inlet and outlet valves close or open; LR_in, out $=0,1$ stand for rear left wheel cylinder inlet and outlet valves close or open; RR_in, out $=0,1$ stand for rear-right wheel cylinder inlet and outlet valves close or open [4].

3.2. The Fuzzy Controller Design. A two-dimensional fuzzy controller is adopted in this paper, and the deviation of the yaw rate of the vehicle $E$ and the deviation rate of the yaw rate $E C$ are the input of the controller. The regulating pressure of differential braking wheel cylinders offside diagonal of the failed tire is the output of the controller.

If we fuzz the input variables and output variables and divide the input variables and output variables into 7 grades on average, then they are "negative big" (NB), "negative middle" (NM), "negative small” (NS), "zero" (ZE), "positive small” (PS), "positive middle" (PM), and "positive big” (PB), respectively [7]. 


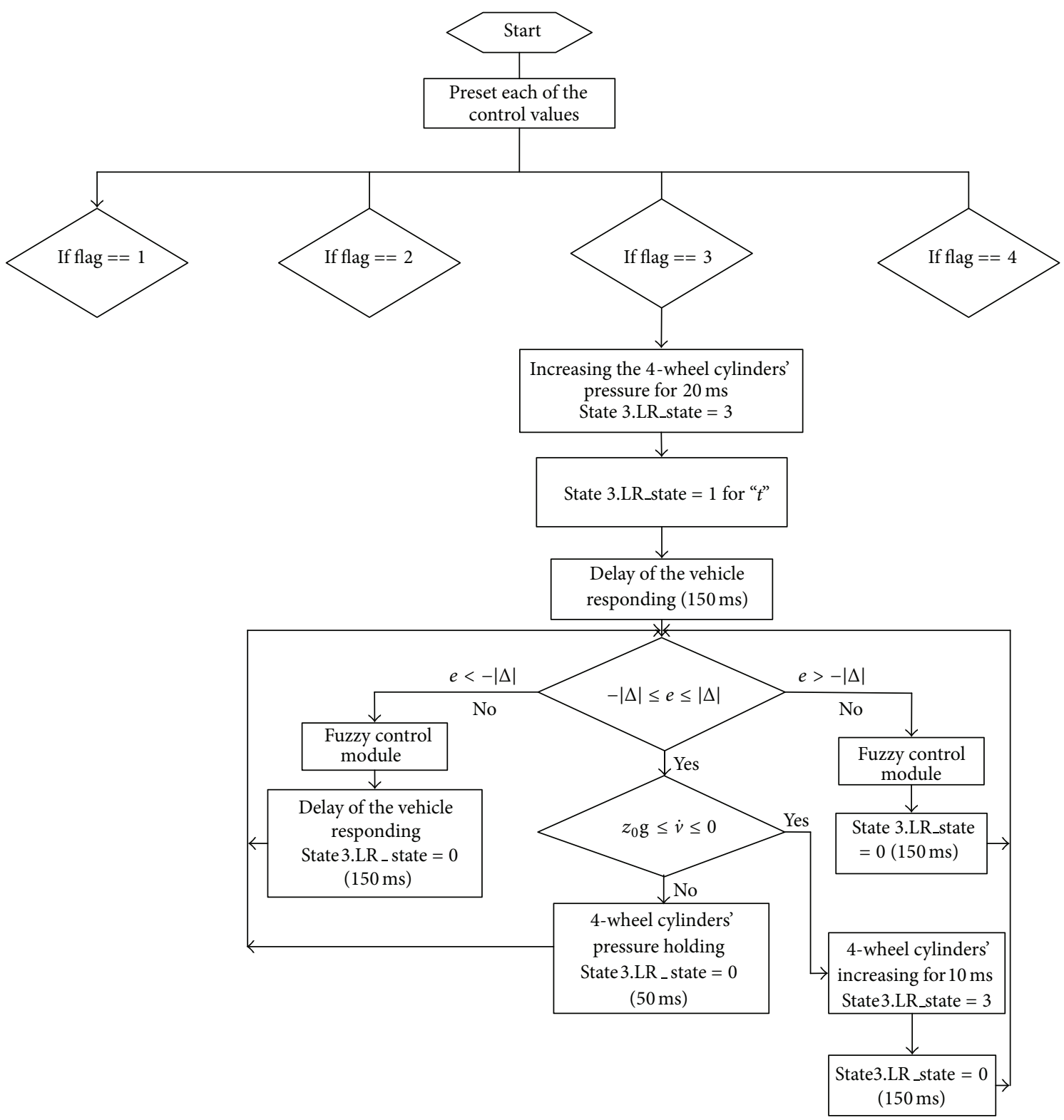

Figure 3: Controlling procedure for tire failure.

The design principle of the fuzzy controller is that when the actual value is far less than the desired value, namely, NB, and the deviation of the changing rate is also far less than the expectations, for NB, in order to eliminate deviation as soon as possible, we need to exert a positive and big yaw torque to the vehicle; namely, we should take the control value as the maximum of "positive big" PB at this moment. As for PS case, if the deviation changing rate is "positive small" PS, and the deviation is in the tendency of decreasing, we should take a positive middle control yaw torque to speed up the target and at the same time to prevent the overregulating of the system. When the deviation comes to PS or PB, it is prone to overregulating, so no control amount or small control amount should be increased at this moment. When the actual value is bigger than the desired state, namely, $\mathrm{PB}$, and the deviation of the changing rate is also bigger than the expectations, in order to eliminate deviation as soon as possible, we need to perform a negative big yaw torque on the vehicle; namely, we should take the control measuring as the maximum of "negative big" NB at this moment. When the deviation is small, in order to prevent overregulating and stabilize the system as soon as possible, the principal contradiction is transformed into stable problems. So we need to regulate the control quantity mainly according to the changing rate of deviation [9]. The fuzzy control rules are listed in Table 1 according to the principle of fuzzy control principle.

The input variables of the controller are the deviation of yaw rate named $E$ and the deviation change rate of yaw rate named $E C$, and the output parameter named $P_{w}$ is the wheel 


State 1.LF_state $=0$
LF_in $=0$ LF_out $=0$
RF_in $=0$ RF_out $=0$
LR_in $=0$ LR_out $=0$
RR_in $=0$ RR_out $=0$
$\mathrm{FB}=1 \mathrm{RB}=1$
$\mathrm{FS}=1 \mathrm{RS}=1$

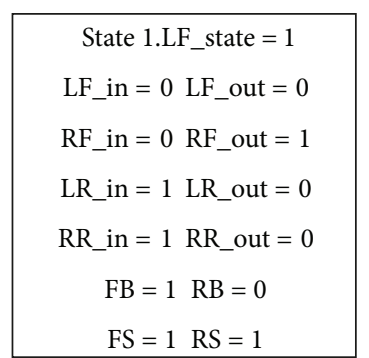

State 1.LF_state $=2$
LF_in $=0$ LF_out $=0$
RF_in $=1$ RF_out $=0$
LR_in $=1$ LR_out $=0$
RR_in $=1$ RR_out $=0$
FB $=1$ RB $=0$
$F S=1$ RS $=1$

a-LF

State 3.LR_state $=0$
LF_in $=0$ LF_out $=0$
RF_in $=0$ RF_out $=0$
LR_in $=0$ LR_out $=0$
RR_in $=0$ RR_out $=0$
FB $=1$ RB $=1$
FS $=1$ RS $=1$

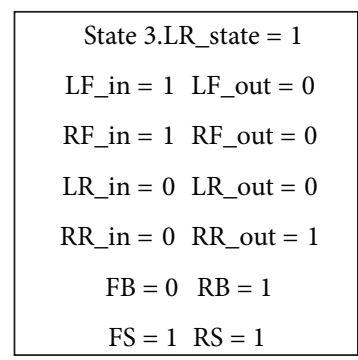
State 3.LR_state $=2$
LF_in $=1$ LF_out $=0$
RF_in $=1$ RF_out $=0$
LR_in $=0$ LR_out $=0$
RR_in $=1$ RR_out $=0$
$\mathrm{FB}=0 \quad \mathrm{RB}=1$
$\mathrm{FS}=1 \quad \mathrm{RS}=1$

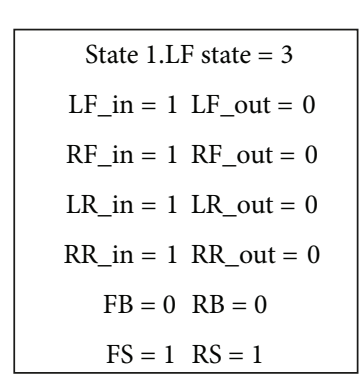

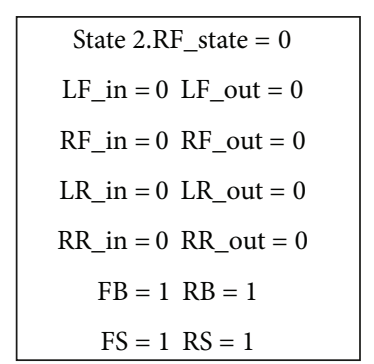

State 2.RF_state $=2$
LF_in $=1$ LF_out $=0$
RF_in $=0$ RF_out $=0$
LR_in $=1$ LR_out $=0$
RR_in $=1$ RR_out $=0$
FB $=1$ RB $=0$
FS $=1$ RS $=1$

b-RF
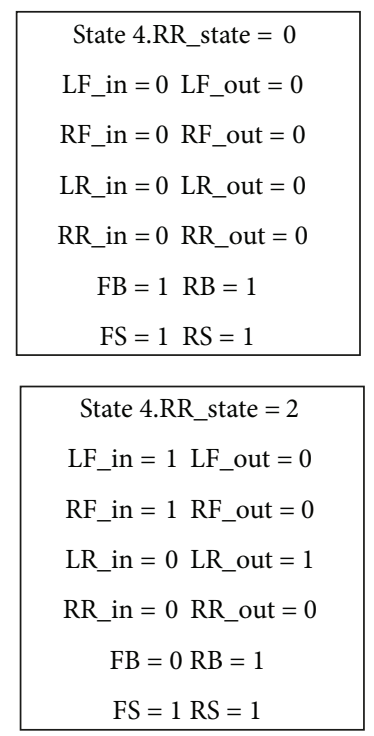
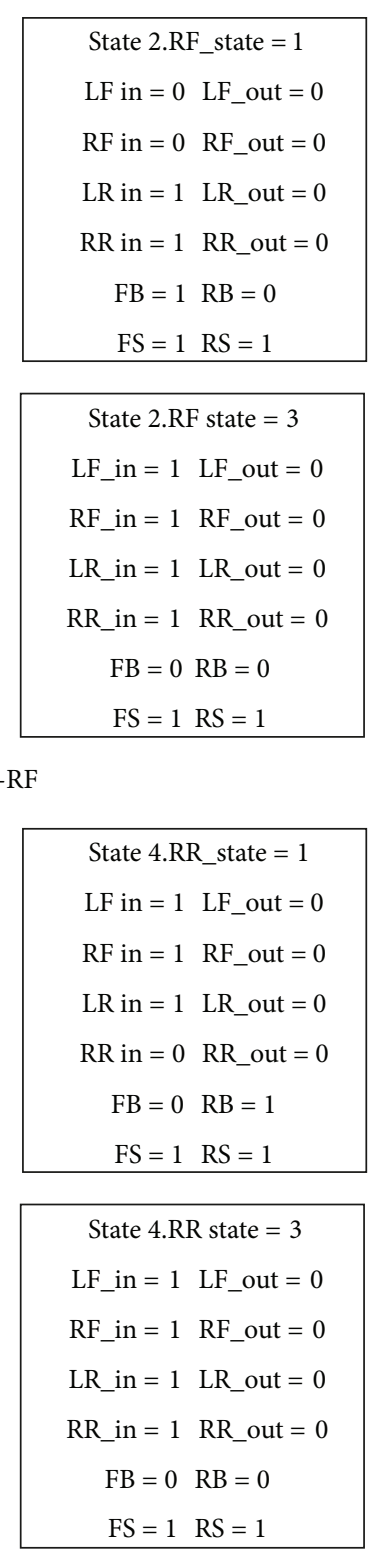

d-RR

FIgURE 4: State value of each tire failure control.

cylinder regulating force of the offside diagonal wheels of the failure tire, where

$$
E=\gamma-\gamma_{\text {ref }}, \quad E C=\dot{\gamma}-\dot{\gamma}_{\text {ref }} .
$$

When the right-front or left-rear wheel tire failure occurs, the cylinder valves offside diagonal of the failure wheel is controlled, and the related fuzzy controlling rules outputs are shown in Table 2. The left-front or right-rear wheel tire failure is opposite to the rules of Table 2, which is shown in Table 3.

\section{Road Test}

Tire blowout device is shown in Figure 5. It includes a receiver, a transmitter, a remote control, an electromagnetic valve, and a tire blowout simulating control valve.
TABLE 1: Fuzzy control rule.

\begin{tabular}{lccccccc}
\hline E & & & & EC & & \\
& NB & NM & NS & ZE & PS & PM & PB \\
\hline NB & PB & PB & PB & PB & PM & PS & ZE \\
NM & PB & PB & PM & PM & PS & ZE & ZE \\
NS & PM & PM & PM & PM & ZE & ZE & NS \\
ZE & PM & PM & PS & ZE & ZE & NS & NM \\
PS & PS & PS & ZE & NM & NM & NM & NM \\
PM & ZE & NS & NS & NM & NB & NB & NB \\
PB & NS & NS & NM & NB & NB & NB & NB \\
\hline
\end{tabular}

The test vehicle is BJ2500. The average air pressure of the tires is charged to normal $(250 \mathrm{kPa})$, and the testing ground is 
TABLE 2: Output between the fuzzy rule and the wheel cylinder valve control of the offside of the failed tire after FR or RL tire blowout.

\begin{tabular}{|c|c|c|c|}
\hline Related state value & Fuzzy rule of $P_{w}$ & State of valve & Duration \\
\hline \multirow{3}{*}{ Statei.i_state $=1$} & $P_{w}(\mathrm{~PB})$ & In $=1$, Out $=0$ & Long \\
\hline & $P_{w}(\mathrm{PM})$ & In $=1$, Out $=0$ & Middle \\
\hline & $P_{w}(\mathrm{PS})$ & In $=1$, Out $=0$ & Short \\
\hline Statei.i_state $=0$ & $P_{w}(\mathrm{ZE})$ & In $=0$, Out $=0$ & Vehicle responding \\
\hline \multirow{3}{*}{ Statei.i_state $=2$} & $P_{w}(\mathrm{NS})$ & In $=0$, Out $=1$ & Short \\
\hline & $P_{w}(\mathrm{NM})$ & In $=0$, Out $=1$ & Middle \\
\hline & $P_{w}(\mathrm{NB})$ & In $=0$, Out $=1$ & Long \\
\hline
\end{tabular}

TABLE 3: Output between the fuzzy rule and the wheel cylinder valve control of the offside of the failure tire after FL or RR tire blowout.

\begin{tabular}{lccc}
\hline Related state value & Fuzzy rule of $P_{w}$ & State of valve & Duration \\
\hline Statei.i_state $=1$ & $P_{w}(\mathrm{~PB})$ & In $=0$, Out $=1$ & Long \\
& $P_{w}(\mathrm{PM})$ & In $=0$, Out $=1$ & Middle \\
& $P_{w}(\mathrm{PS})$ & In $=0$, Out $=1$ & Short \\
\hline Statei.i_state $=0$ & $P_{w}(\mathrm{ZE})$ & In $=0$, Out $=0$ & Vehicle responding \\
\hline & $P_{w}(\mathrm{NS})$ & In $=1$, Out $=0$ & Short \\
Statei.i_state $=2$ & $P_{w}(\mathrm{NM})$ & In $=1$, Out $=0$ & Middle \\
& $P_{w}(\mathrm{NB})$ & In $=1$, Out $=0$ & Long \\
\hline
\end{tabular}

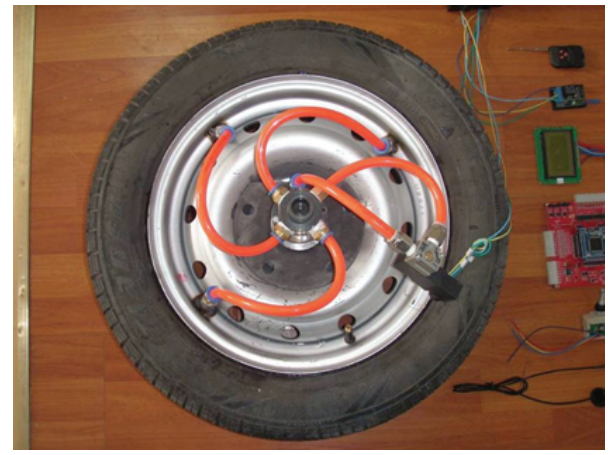

FIGURE 5: The tire blowout device.

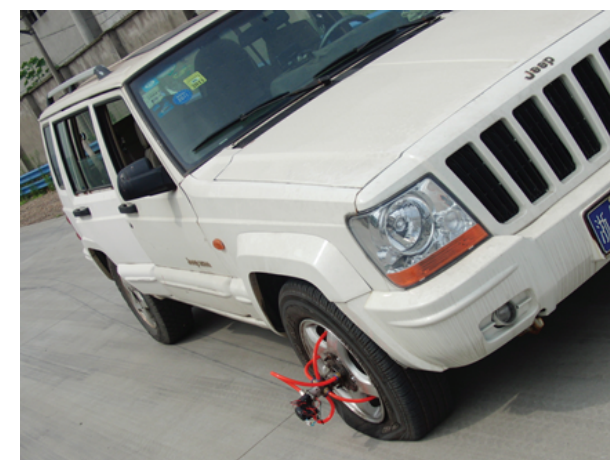

FIGURE 6: Road test set. a flat cement road (a vehicle braking system testing ground of a company). The tire blowout device is controlled by a remote switch. To detect the changes of speed reducing during the braking process of the tire blowout in the road test system, an accelerate sensor is installed near the center of mass of the vehicle. The road test set is shown in Figure 6. The yaw rate signal, longitudinal accelerate signal, and wheel velocity signal are collected by DL750 oscilloscope through which the state of longitudinal driving and yaw rate responding of vehicle are recorded.

The oscilloscope mainly collects three ways of signal including trigger signal $(\mathrm{CH} 1)$, yaw rate signal $(\mathrm{CH} 2)$, and vehicle accelerating signal $(\mathrm{CH} 3)$. With this test set, we can simulate the processing of the vehicle tire blowout. After turning on the remote controller, the electromagnetic valve works, and the air in the tire blows out from four pipes simultaneously. At the same time, the signal of tire blowout is sent to the emergency braking system. Certainly, there is time delay for identification of tire blowout, so we need to give an estimated delay time for the tire blowout signal.

Figure 7 is the outputs of the yaw rate and acceleration when the vehicle speed is $60 \mathrm{~km} / \mathrm{h}$ without stability control after the tire failure. Figure 8 is the outputs of the yaw rate and acceleration when the vehicle speed is $60 \mathrm{~km} / \mathrm{h}$ with the control of stability control strategy of automatically braking after tire failure.

From Figure 7, we can see that when there is no stability control, the fluctuation magnitude of the yaw rate is high in about two seconds after tire failure, then the yaw rate drops to about 2.25, together with the decreasing of the vehicle speed (in 1.5 seconds), and the yaw rate comes back to the basic value (2.5). The acceleration signal fluctuates in about two seconds after tire failure, and then it decreases at a certain speed, with the steady deceleration time about 2 seconds.

From Figure 8, we can see that when there is stability control, the yaw rate fluctuates in about two seconds after tire failure, but the fluctuation magnitude is much lower than 


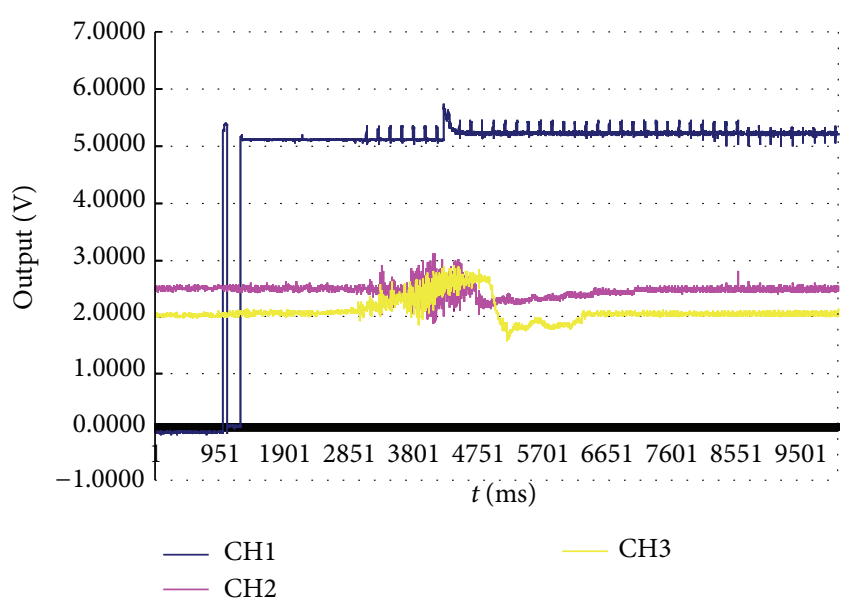

FIGURE 7: The response of vehicle yaw rate and acceleration without tire blowout stability control at $60 \mathrm{~km} / \mathrm{h}$.

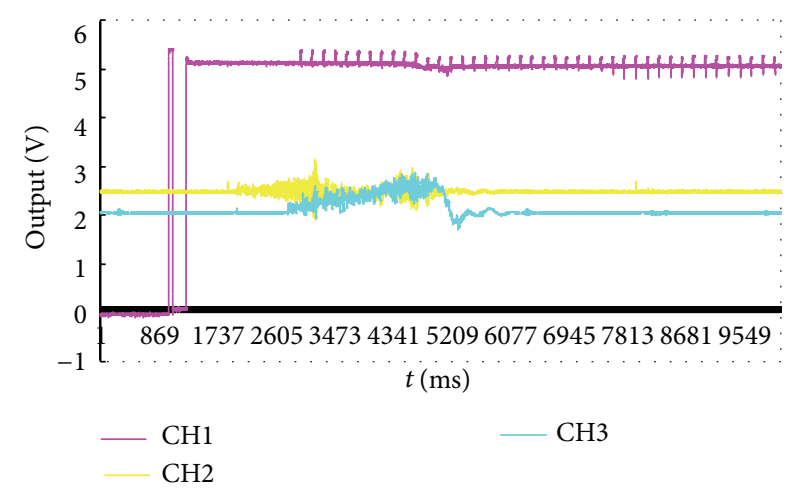

FIGURE 8: The response of vehicle yaw rate and acceleration with tire blowout stability control at $60 \mathrm{~km} / \mathrm{h}$.

that without stability control, and then it basically comes back to the original value until the vehicle almost stops. The acceleration signal fluctuates within more than two seconds after tire failure, and the deceleration time is basically equal to that without stability control, and the deceleration time coming back to stable is shorter than that without the stability control.

Also, from Figure 7, we can see that after triggering time, that is, tire blowout occurring, the yaw movement of the vehicle occurred at about $2200 \mathrm{~ms}$ later. With this time, we can apply the brakes to the vehicle first and then control its yaw movement with our emergency automatic braking system shown in Figure 8.

\section{Conclusions}

The tire blowout emergency automatic braking system produces an additional yaw torque to resist the tire failure yaw movement, in order to maintain the driving track of the vehicle after the tire failure. Once the vehicle deviates from the original trajectory, the fuzzy control strategy is activated, and it adjusts the trajectory by the differential braking until the vehicle comes down to a safe speed.

The tests show that with the action of this system, it can help to slow down the vehicle at a target braking intense and, at the same time, stabilize the tire-failure vehicle quickly to maintain the original track.

\section{Conflict of Interests}

The authors declare that there is no conflict of interests regarding the publication of this paper.

\section{Acknowledgments}

This work was supported by "The Natural Science Foundation" (BK2011367) and "Six Peak Talents Foundation" (SZ2010002) of Jiangsu Province funded by the Chinese Government.

\section{References}

[1] F. Jianzhong and S. Yong, "The tyre pressure monitoring and vehicle deceleration system for tire blow-out," Automobile Engineering, vol. 28, no. 2, pp. 199-200, 2006.

[2] X. lina, X. Jianfeng, and S. Jinhao, "The tire burst of automotive early-warning system design," in Proceedings of the International Conference on Networks Security, Wireless Communications and Trusted Computing (NSWCTC '09), vol. 2, pp. 802-804, April 2009.

[3] S. Patwardhan, M. Tomizuka, W.-B. Zhang, and P. Devlin, "Theory and experiments of tire blow-out effects and hazard reduction control for automated vehicle lateral control system," in Proceedings of the American Control Conference, pp. 12071209, July 1994.

[4] Z. Lozia, "Simulation and test of biaxial vehicle motion after a 'Tire Blow-out,' SAE Paper, 2005, 010410.

[5] D. Lu, X. Song, and F. Tao, The Research Report of Dynamic Characteristics Tests of Blow-Out Tire, State Key Laboratory of Automotive Dynamic Simulation, Changchun, China, 2010.

[6] Q. Chen, "Research on stability control of vehicle emergency braking when tire blow-out," Postdoctoral Report, Zhejiang Asia-Pacific Mechanical \& Electronic Co., Ltd, Hangzhou, China, 2011.

[7] J. Huang, K. Guo, X. Song, and W. Liu, "Vehicle stability control method after tire blow-out," China Mechanical Engineering, vol. 20, no. 16, pp. 2006-2007, 2009.

[8] R. Robinette, D. Deering, and R. J. Fay, "Drag and steering effects of under inflated and deflated tires," SAE Paper, 970954.

[9] S. Velupillai and L. Güvenç, "Tire pressure monitoring," IEEE Control Systems Magazine, vol. 27, no. 6, pp. 22-25, 2007. 

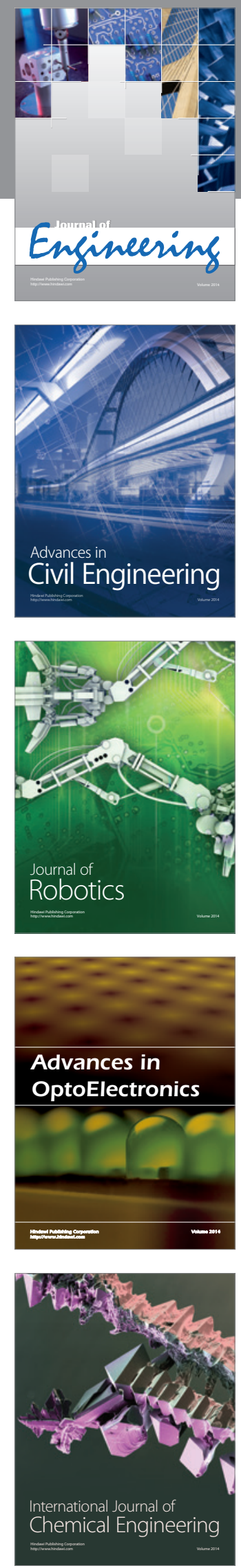

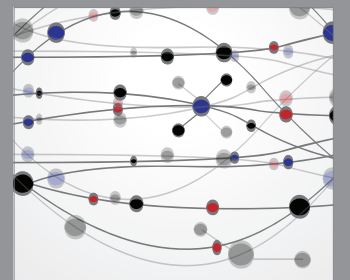

The Scientific World Journal
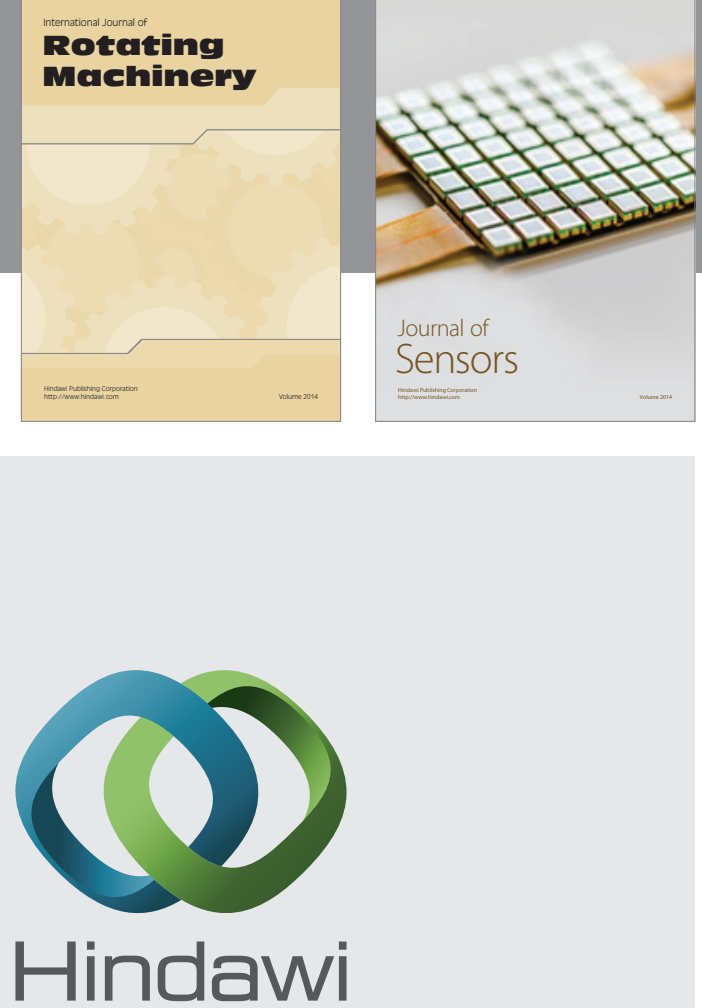

Submit your manuscripts at http://www.hindawi.com
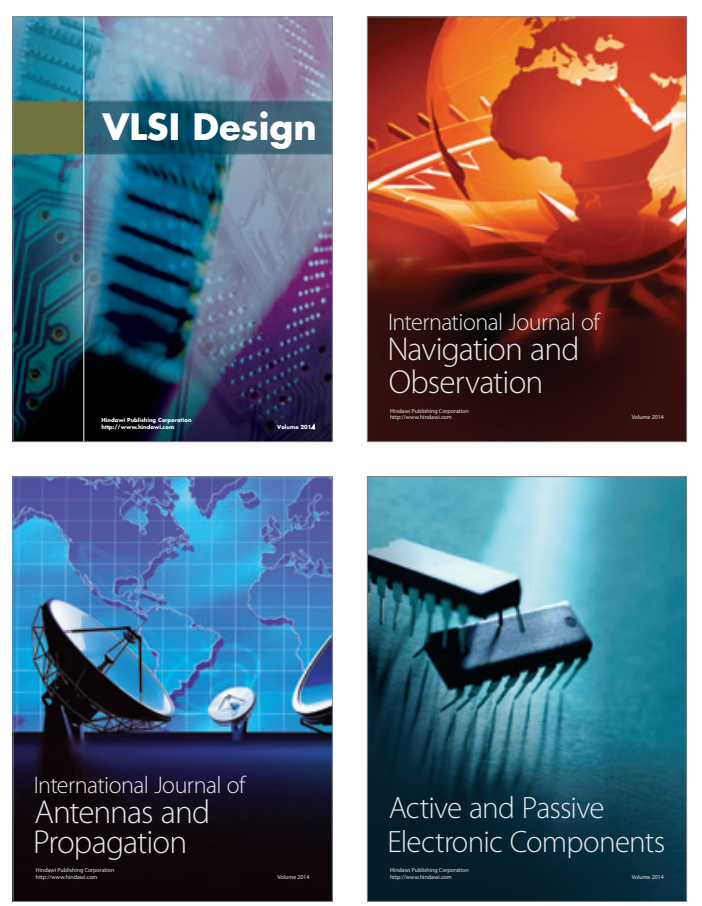
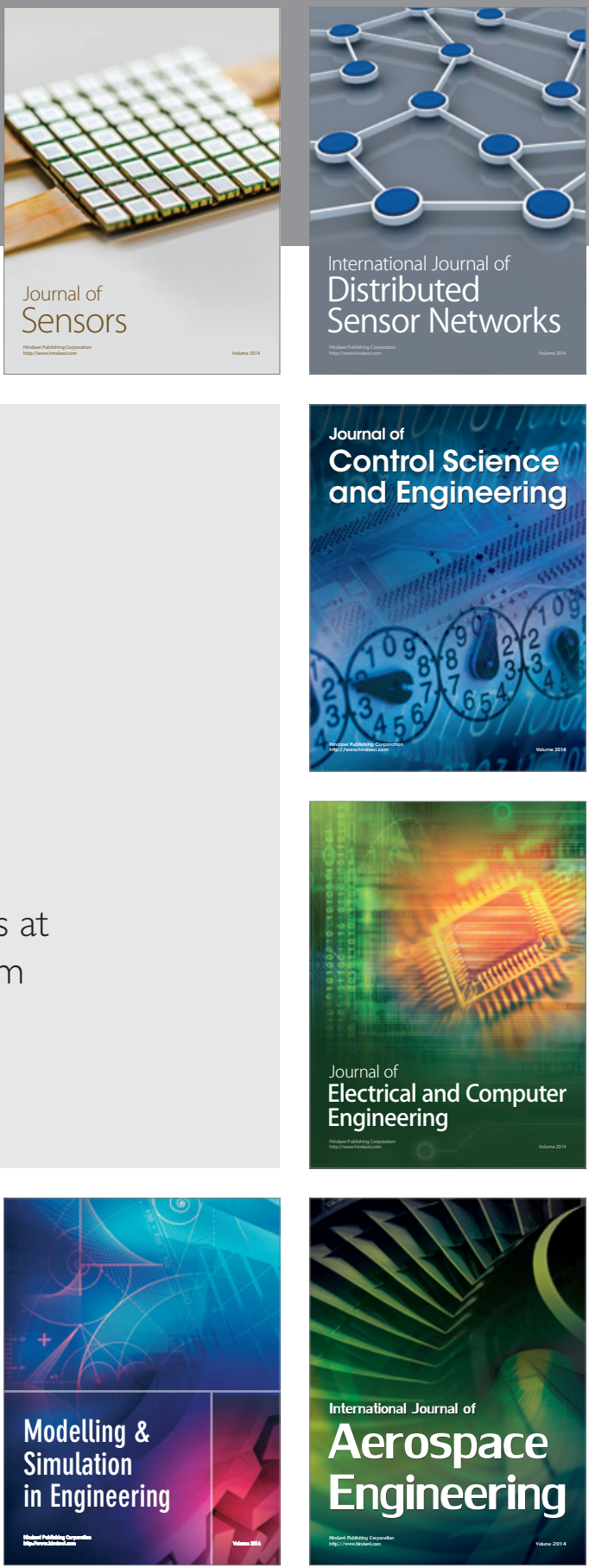

Journal of

Control Science

and Engineering
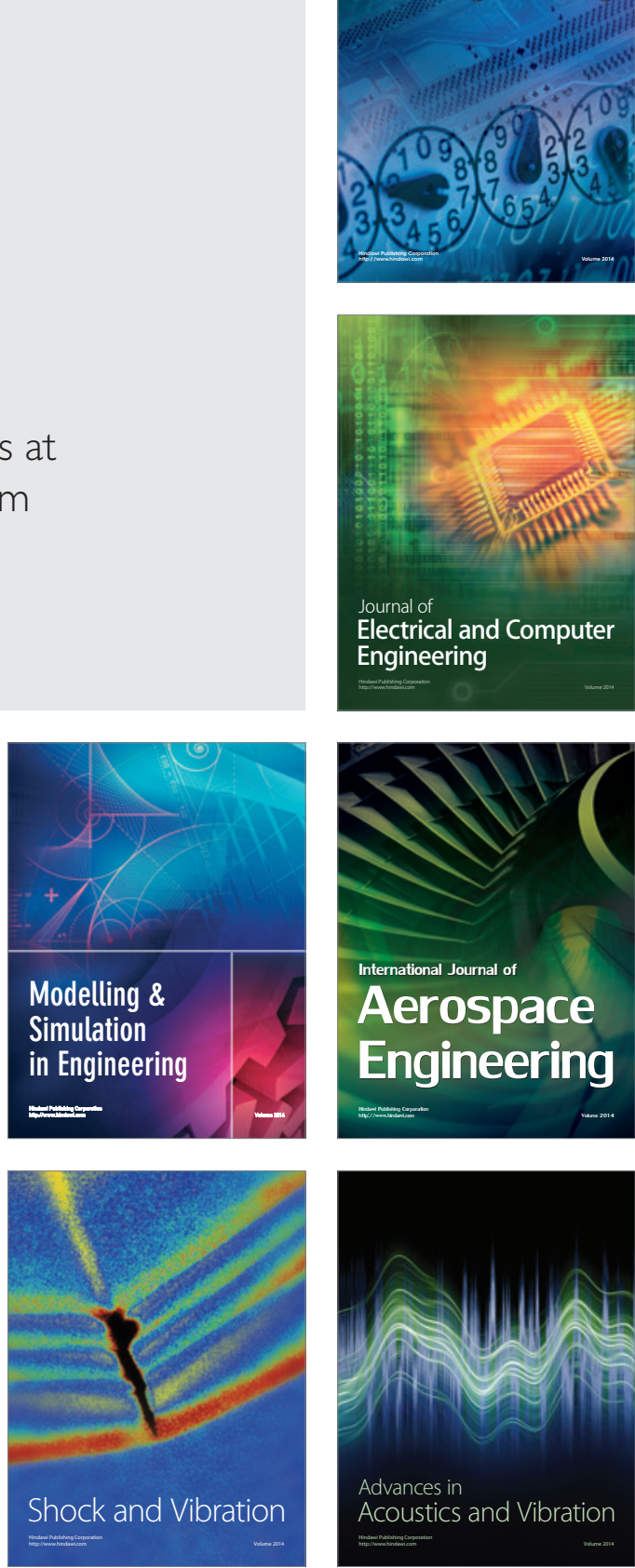